\title{
Young-Dentist Frühjahrsmeeting in Bonn - wieder ein voller Erfolg!
}

\begin{abstract}
Am letzten Aprilwochenende trafen sich erneut junge angehende Zahnmedizinerinnen und Zahnmediziner, um gemeinsam im Gustav-Stresemann-Institut in Bonn beim Frühjahrsmeeting der young dentists (yd2) betriebswirtschaftliche und fachliche Vorträge zu genießen. Die young dentists können als Nachwuchsinitiative des Freien Verband Deutscher Zahnärzte (FVDZ) und der Akademie Praxis und Wissenschaft (APW) auf ein umfangreiches Know-how zurückgreifen, um als junge Kolleginnen und Kollegen in ihrem beruflichen Werdegang unterstützt zu werden.
\end{abstract}

$\mathrm{D}$ er Freitagnachmittag begann mit dem betriebswirtschaftlichen Vortrag „Abrechnung, Abdingung und freie Vertragsgestaltung von außervertraglichen Leistungen beim GKVund PKV-Patienten“. Referentin Irmgard Marischler überzeugte die Teilnehmer in ihrem Vortrag, dass Aufklärung- und Dokumentationspflichten nicht nur notwendig sind, sondern darüber hinaus zu einer Zufriedenheit bei der Honorierung führen, wenn man genau weiß, welche Möglichkeiten in der Rechnungsstellung erlaubt sind und wie sie genutzt werden dürfen.

Auch beim gemeinsamen Abendessen mit Referentin Marischler wurde neben dem gemütlichen Miteinander noch die Möglichkeit genutzt, offene Fragen zu klären, für welche die Zeit im Vortrag nicht mehr gereicht hatte.

\section{Neue Restaurationstechnik im Fokus}

Restaurative Zahnheilkunde stand dann am Samstag im Fokus. Hier zeigten die Heidelberger Referentinnen PD Dr. Cornelia Freese, PD Dr. Diana Wolff und ZÄ Theresa Friederike Wohlrab, was mit der richtigen Komposittechnik heute bereits alles möglich ist. So stellte Freese in ihrem Vortrag „Die Versorgung extrem tiefer Kavitäten mittels neuartiger Restaurationstechniken“ die zweiphasige direkte Restaurationstechnik (R2-Technik) vor und erklärte, warum sie bei richtiger Indikationsstellung eine Alternative zum klassischen chirurgisch-prothetischen Vorgehen ist.

Wolff ermutigte die Teilnehmer in Ihrem Vortrag „Mehr Zahnerhaltung wagen - die unbegrenzten Möglichkeiten der direkten Komposittechniken“ anhand zahlreicher klinischer Beispiele, viel häufiger substanzschonende Restaurationen mittels Komposit zu realisieren. Hierzu stellte Sie die richtigen Wege und Hilfsmittel vor, um ästhetisch und funktionell perfekte Ergebnisse zu erzielen.
Dass diese zusätzlichen Möglichkeiten der Kompositanwendung wissenschaftlich fundiert sind, zeigte Z $\mathrm{A}$ Wohlrab in Ihrem Vortrag „Gibt es Evidenz für die erweiterten Indikationsbereiche der direkten Komposittechniken?"

Vielen Dank an dieser Stelle nicht nur an die Referentinnen sondern auch an die Mitarbeiter/-innen vor Ort von Straumann und der ZA.

Dr. Christian Honert

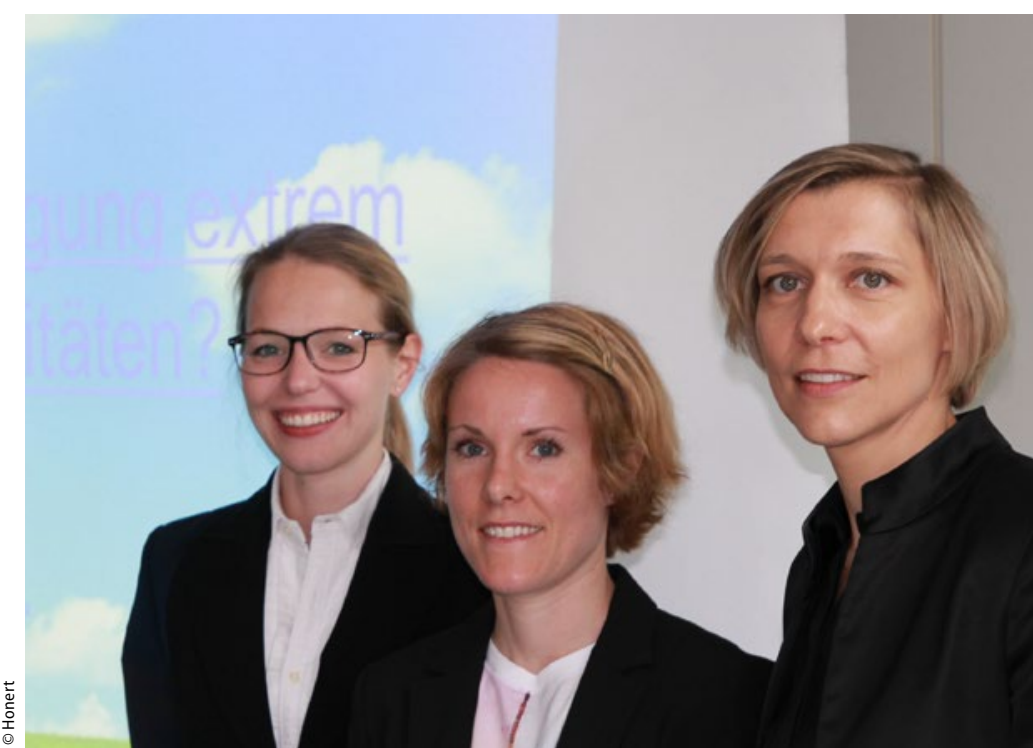

Die dei Expertinnen aus Heidelberg: Theresa Wohlrab,

PD Dr. Cornelia Freese und PD Dr. Diana Wolff infomierten über neue Komposittechniken
- Hirschfeld: Meinen Forschungsaufenthalt habe ich in Boston (USA) am Forsyth Institute, im Department of Immunology and Infectious Diseases, absolviert. Dieses renommierte Institut beschäftigt sich im Schwerpunkt mit zahnmedizinischer Grundlagenforschung, hat in der Vergangenheit bereits mit wichtigen Erkenntnissen zum Verständnis parodontaler Erkrankungen beigetragen und war daher der ideale Ort. Ich habe auch weiterhin die Möglichkeit, dort Teilprojekte zu bearbeiten oder Experimente durchzuführen sowie auf die Expertise der Mitarbeiter zurückzugreifen. Des Weiteren habe ich dort nicht nur neue Kollegen, sondern auch neue Freunde getroffen, zu denen der gute Kontakt sicher noch lange bestehen bleiben wird.

Brakel: Frau Dr. Hirschfeld, wir danken Ihnen für das Gespräch.

Weitere Informationen zum Haase-Stipendium finden sich auf der DGZMK-Homepage www.dgzmk.de unter der Rubrik „Wissenschaftliche Preise". 